\title{
Acústica de los órganos de tubulares y tendencias futuras en la investigación
}

\author{
Judit Angster ${ }^{A}$, Péter Rucz ${ }^{B}$, András Miklós ${ }^{A}$ \\ ${ }^{A}$ Fraunhofer Institute for Building Physics IBP \\ B Budapest University of Technology and Economics \\ judit.angster@ibp.fraunhofer.de
}

\section{RESUMEN}

En este trabajo se presentan explicaciones cientificas para tener un mejor entendimiento de cómo funcionan los órganos tubulares y revisar algunas creencias comunes en su construcción, buscando mejorar este arte.

\section{PALABRAS CLAVE}

Órganos tubulares, acústica de los órganos de tubo.

\begin{abstract}
Scientific explanations for having a better understanding of the operation of pipe organs, and revision of some common beliefs regarding their building, seeking to improve this art, are presented in this work.
\end{abstract}

\section{KEYWORDS}

Organ pipes, acoustics of the organ pipes.

\section{INTRODUCCIÓN}

El órgano tubular (en adelante órgano) produce un sonido majestuoso que difiere de los otros instrumentos musicales. Debido a su amplia gama tonal, su capacidad de imitar el sonido de varios instrumentos, y su gran tamaño, el órgano es llamado a menudo el rey de los instrumentos musicales (figura 1). La riqueza y variedad del color sonoro (timbre) producido por un órgano es muy singular debido a las posibilidades casi incontables para mezclar los sonidos de diferentes tubos. De acuerdo con el arte de la generación de sonido, hay dos tipos de tubos en el órgano que son similares en función a otros instrumentos de viento: flauta (labial) y de lengüeta. Aunque este artículo se centra en la excitación sonora por los tubos, se menciona brevemente el papel de los tubos de lengüeta (figura 2). El artículo también muestra cómo se puede entender la conexión entre el carácter sonoro y la forma y dimensiones de los tubos, así como las tendencias en la investigación que se centran en ayudar a los constructores de órganos en su trabajo práctico.

\section{ESTRUCTURA DEL ÓRGANO TUBULAR}

La figura 3 muestra un bosquejo de un órgano de tubos. Sus partes principales son el cofre de viento con los tubos, el sistema de viento y el sistema de control (teclado, conexiones mecánicas y topes; figura 2). Los tubos se organizan en la 

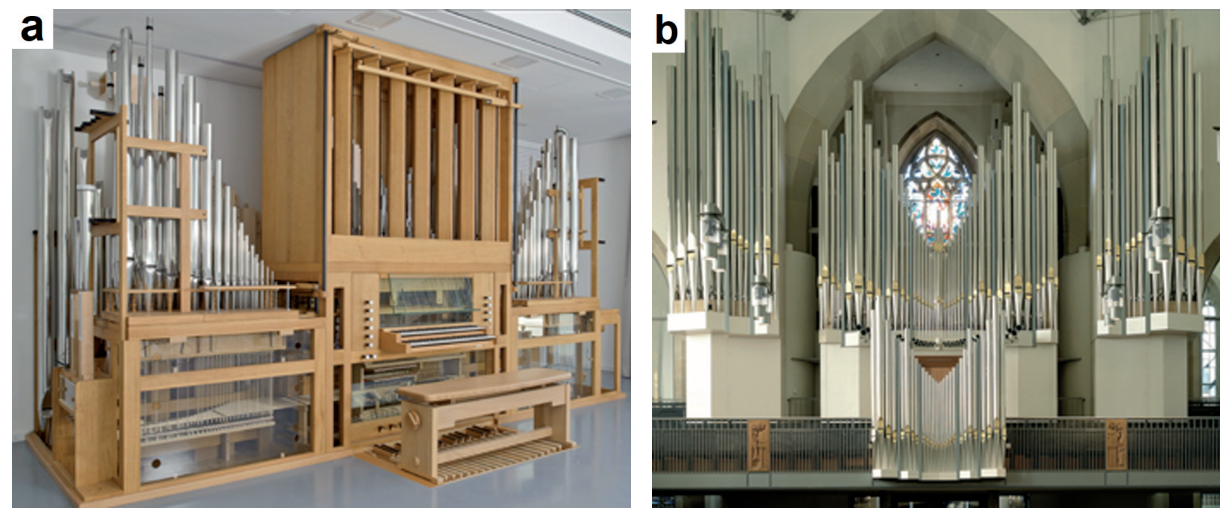

Fig. 1. a: Órgano de investigación en el Fraunhofer Institute of Building Physics IBP en Stuttgart, Alemania, construido por Mühleisen (Leonberg, Alemania) en 2011. Los pedales se pueden ver bajo el banco. Foto de Roman Wack. b: Órgano de tubos en el Stiftskirche en Stuttgart, Alemania, construido por Mühleisen (Leonberg, Alemania) en 2004. Los tubos frontales se pueden ver claramente. Foto de Theo Holder.

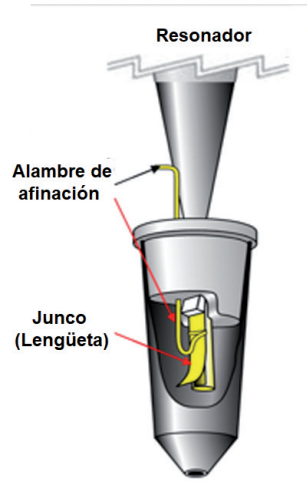

a)

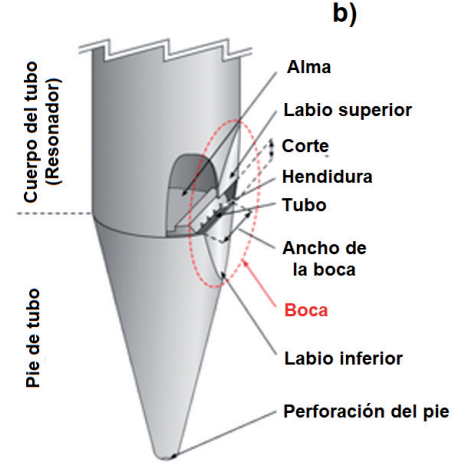

Fig. 2. Las partes de la caña (lingual; a) y un pasaje (labial; b) de un órgano de tubos. Como se muestra en $b$, el corte es la distancia entre el labio inferior y superior.

caja del viento según la nota y el timbre. Un conjunto de tubos que producen el mismo timbre para cada nota se llama un rango y cada tecla en un órgano de tubo controla una nota que puede ser tocada por diferentes rangos de tubos, solo o en combinación (ver http://acousticstoday.org/organ_stop; para una demostración, véase http://acousticstoday.org/rank).

Los rangos pueden ser activados por un tope. El deslizador (figura 3) consiste en una tabla de madera que tiene una serie de agujeros que se hacen coincidir con los tubos colocados en una fila. Al activar uno de los topes, los agujeros de la placa deslizante dejan pasar el flujo de aire desde el canal de tono en los tubos que tienen el mismo timbre. Todos los órganos producen sonido por medio del aire que fluye en el tubo de modo que cada sonido "consume" cierta cantidad de aire. Un arreglo mecánico conecta el teclado y los pasos de tono en la caja de viento que es también el sistema de conmutación. Cuando se presiona una tecla, la válvula correspondiente en la consola se abre y el aire fluye en el canal de tono y los tubos seleccionados por los topes. Un muelle cierra la válvula, bloqueando el paso del aire cuando se libera la tecla. El aire presurizado es proporcionado por el sistema de viento que consiste de cuatro partes esenciales. (1) El soplador (ventilador eléctrico) que suministra el aire al instrumento. El soplador bombea el aire en el sistema de viento de acuerdo con el consumo del instrumento. 


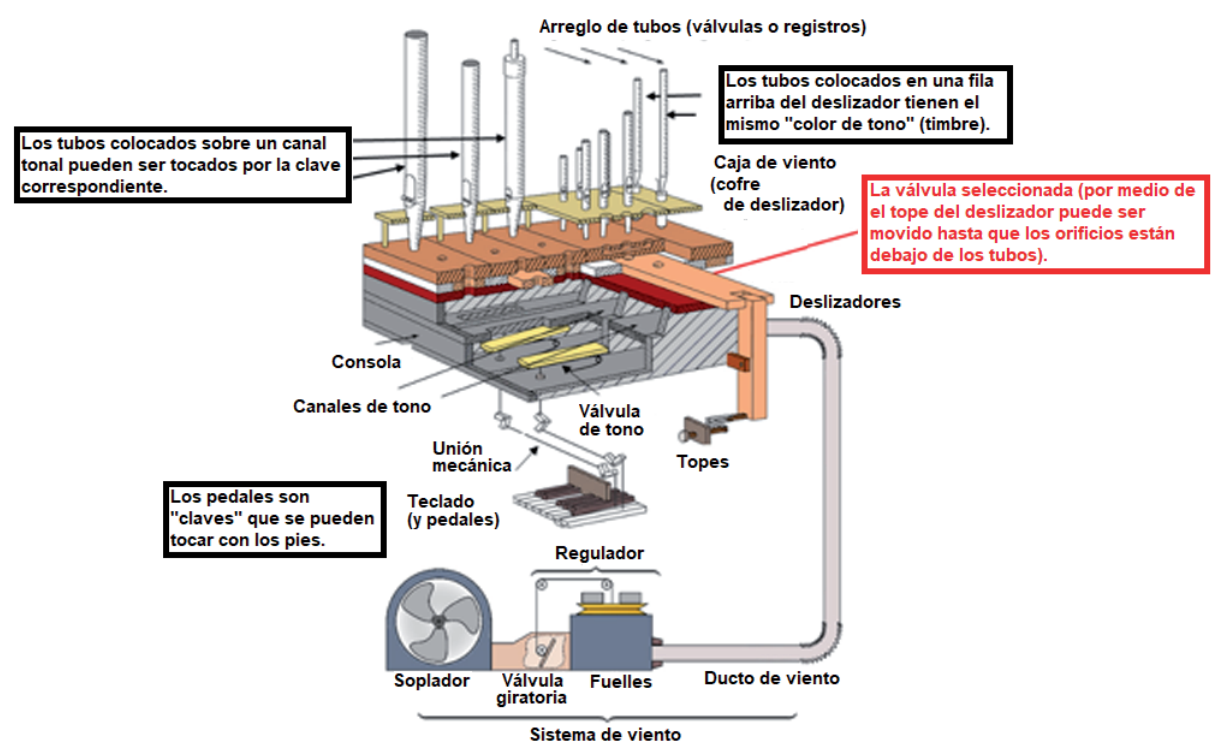

Fig. 3. Un bosquejo de un órgano de tubo y sus partes más importantes.

(2) La válvula giratoria regula el flujo de aire del ventilador en los fuelles. (3) Los fuelles garantizan que la presión en el caja del viento permanece constante.

La presión requerida en el órgano se establece por el constructor colocando pesos en la parte superior de los fuelles. (4) Finalmente, el conducto de viento conecta el sistema de viento con la caja (parte inferior), suministrando el aire a los tubos. Múltiples sistemas de viento pueden estar presentes y operar al mismo tiempo en órganos grandes, pero cada uno proporciona aire a un conjunto diferente de rangos. Como ya se mencionó, hay dos tipos de tubos que son similares en función a otros instrumentos de viento: flauta (labial) tubos (como una flauta o una flauta transversal) y de junco (lingual) (como un clarinete o un saxofón). El sonido del tubo es producido por una banda de latón vibrante conocida como lengüeta. El aire bajo presión (viento) se dirige hacia la lengüeta que vibra en un tono específico. Esto contrasta con la flauta, que no contiene partes móviles y producen sonido sólo a través de la vibración del aire (véase la figura 2). En un órgano típico hay considerablemente más tubos de flauta que de lengüeta. Las partes principales los tubos de junco y de flauta se muestran en figura 2. En las siguientes secciones se discute la física de los tubos. La discusión se basa en una publicación anterior ${ }^{1}$ complementada por algunos resultados de proyectos de investigación europeos llevados a cabo en cooperación con varias empresas constructoras de órganos. Los juncos no se discuten en este documento, pero se pueden consultar en ${ }^{2-4}$.

\section{Flautas}

Aunque las principales características del sonido de los tubos de la flauta se han investigado exhaustivamente, ${ }^{5-7}$ la conexión entre el carácter sonoro, la forma del tubo y las dimensiones todavía no se entienden bien. Sin embargo, en la tradición de construcción de órganos, el carácter sonoro de los diferentes rangos se asocia inequívocamente con la forma de los tubos, el material, y tamaño. ${ }^{8,9}$ Aunque el timbre, y especialmente el ataque de los tubos (justo al 
principio del sonido del tubo), pueden ser cambiados significativamente por los ajustes de voz (cambiando los parámetros geométricos de la tubería como el diámetro de la anchura del conducto y el corte de la boca [labio superior]), las características principales del sonido son bastante estables para un de rango y depende principalmente de la forma y el progreso de dimensiones con nota (escala) de los tubos. Es interesante para los científicos que sólo un rango muy estrecho de todas las dimensiones posibles (diámetro, espesor de pared, altura de corte, ancho de salida) y los materiales se utilizan realmente para tubos de órganos. Algunas de estas limitaciones pueden explicarse por razones técnicas, pero la mayoría de ellas no tienen base en la ciencia.

\section{Resultados experimentales}

Aunque los tubos de flauta ofrecen una gran variedad de sonidos, las propiedades medidas de esos sonidos contienen varios elementos comunes que se pueden utilizar para caracterizarlos. Para determinar estas características se utilizan tres mediciones: el espectro estacionario (el espectro del sonido de un tubo de sondeo continuo) tanto en la boca como en final y el ataque transitorio en la boca. Para hacer esto, los espectros estacionarios se miden por micrófonos colocados cerca $(\sim 3-5 \mathrm{~cm})$ a las dos aberturas de las tuberías y el ataque Los transitorios en la boca se analizan mediante un programa informático especial. ${ }^{10}$

\section{Características destacadas del sonido estacionario y fenómenos físicos relacionados}

El espectro sonoro y las características destacadas de un tubo se pueden ver en la figura 4.

Las filas de tubos de flauta se dividen en tres grupos según a su sonido característico. Los tubos más anchos producen los tonos más fundamentales y los menos armónicos, y comienzan a sonar más rápido (ataque rápido). El Diapasón o la familia principal produce el sonido característico del órgano y no tiene la intención de imitar cualquier otro instrumento o sonido. Son de media escala y son destacadas a menudo en las fachadas de los órganos. Se pueden caracterizar por su fuerte segundo parcial, especialmente en el ataque. Los tubos tan delgados que parecen cuerdas son los más estrechos. Producen un sonido brillante que es bajo en armónicos fundamentales y rico en parciales superiores. Uno de los controles más comunes de estos tubos se llaman Salicional. Los controles siguen a menudo la nomenclatura de los instrumentos cordófonos de arco, como el violoncello, la viola de Gamba, y el Geigen (del alemán Geige, para violín; véase http://acousticstoday.org/flue). Tienen sonidos muy brillantes con más de 20 parciales armónicas pero con un ataque lento. ${ }^{1}$ Se pueden enumerar las características de los espectros sonoros de un órgano y los fenómenos físicos relacionados puede explicarse de la siguiente manera.

\section{Una serie de parciales armónicas}

Es bien conocido por los elementos de la teoría de Fourier ${ }^{11}$ que el espectro de una señal periódica contiene una serie de componentes armónicos (parciales). Estas parciales se pueden ver en la figura 4. 


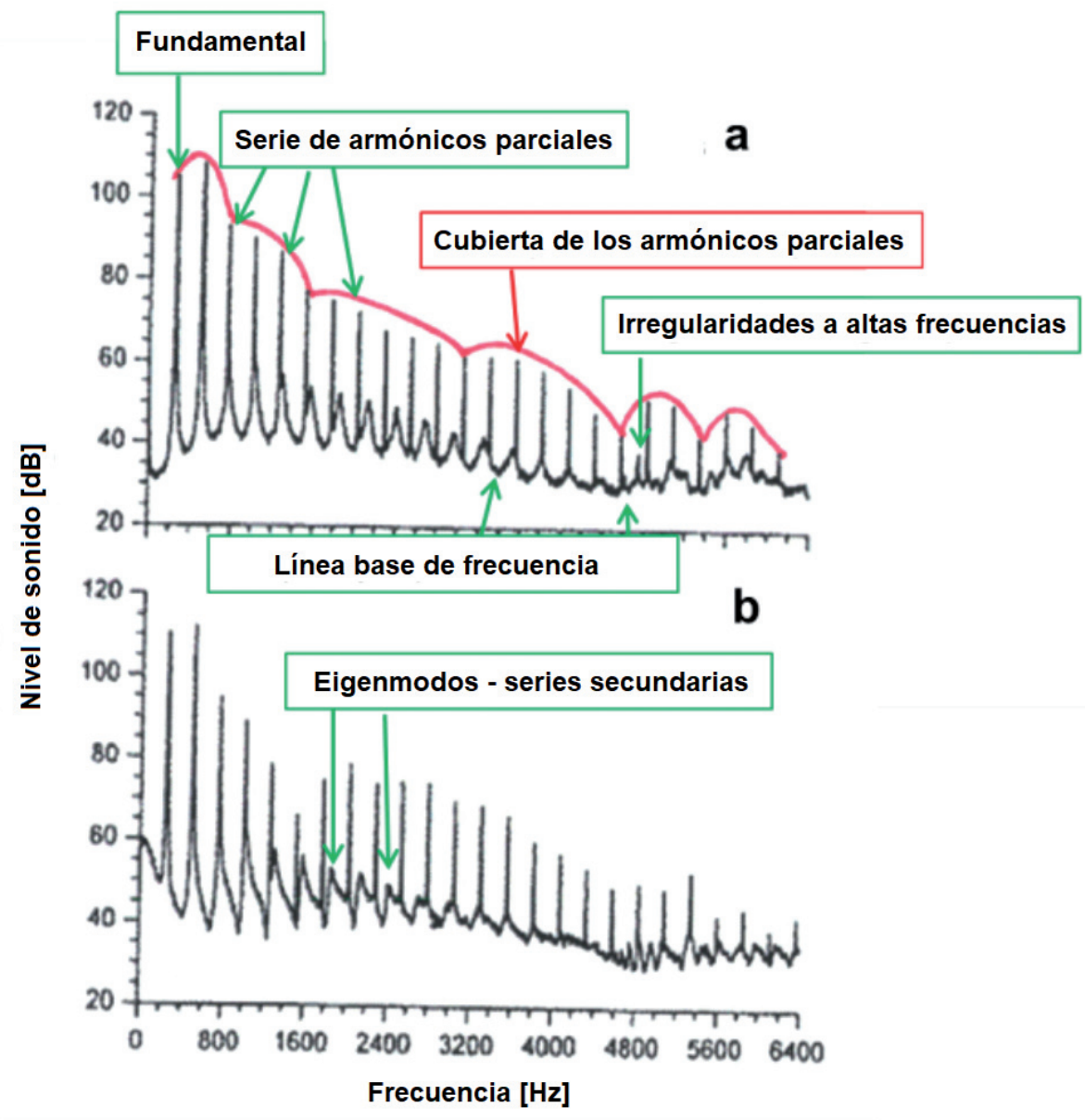

Fig. 4. Espectro estacionario típico de un tubo de órgano de salida en el extremo abierto (a) y en la boca (b). Ver el texto para más detalles.

\section{Una segunda serie de picos más pequeños y más amplios que no están relacionados armónicamente, pero están ligeramente estirados en frecuencia}

Los picos pequeños y anchos mostrados en el espectro demuestran la presencia de eigenmodos acústicos (ondas estacionarias) de los tubos. (Se le llama onda estacionaria a aquella en que las ondas sonoras reflejadas de ida y vuelta en el tubo se combinan de tal manera que cada ubicación a lo largo del eje tiene una amplitud constante pero diferente. Las ubicaciones las amplitudes mínimo y máxima se llaman nodos y antinodos, respectivamente. La frecuencia de la onda estacionaria es la frecuencia de resonancia o frecuencia eigenfrequency del tubo. Las ondas estacionarias se producen en un tubo en varias frecuencias). La presencia de eigenmodos puede ser probada experimentalmente mediante el uso de excitación acústica externa. Si se coloca un tubo en el camino del sonido generado por un altavoz, el tubo amplificará los componentes de frecuencia que corresponden a las eigenresonancias. El espectro de eigenresonancia puede ser determinado colocando un pequeño micrófono en el tubo y usando una excitación en un rango amplio de frecuencia. Tal espectro se muestra en la figura 5 para un tubo cilíndrico. 


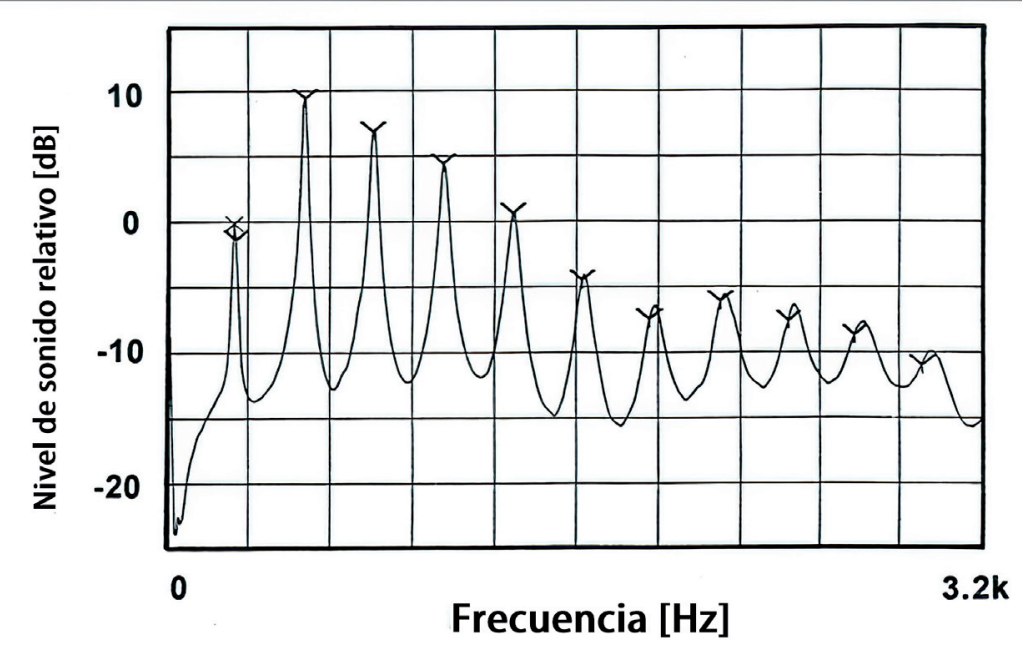

Fig. 5. Características de un tubo de $60 \mathrm{~cm}$ de largo y $31 \mathrm{~mm}$ de diámetro. Las partículas armónicas están marcadas por cursores en forma de " $v$ ".

Las eigenresonancias están ligeramente estiradas; las eigenfrequencias son un poco más altas que los armónicos de la primera eigenresonancia. El estiramiento de las frecuencias propias es mucho más pronunciado en los tubos de órganos abiertos. En el espectro de un tubo Diapasón (figura 4a), la novena eigenresonancia se encuentra a medio camino entre el noveno y décimo armónico parcial. El estiramiento se hace más grande para relaciones de diámetro-longitud más grandes y para aberturas más pequeñas en los extremos del tubo. La distribución espacial medida de los modos primer, tercero y quinto tubo de flauta bastante ancho se muestra en la figura 6 . Se puede observar que las ondas estacionarias caen asimétricamente en el tubo; desplazadas hacia la boca. Además, la media longitud de onda del primer modo eigenmodo (y $\mathrm{n}$ veces la media longitud de onda del enésimo eigenmodo) es más largo que la longitud del resonador. La diferencia puede considerarse como una "corrección final" para los cálculos prácticos.

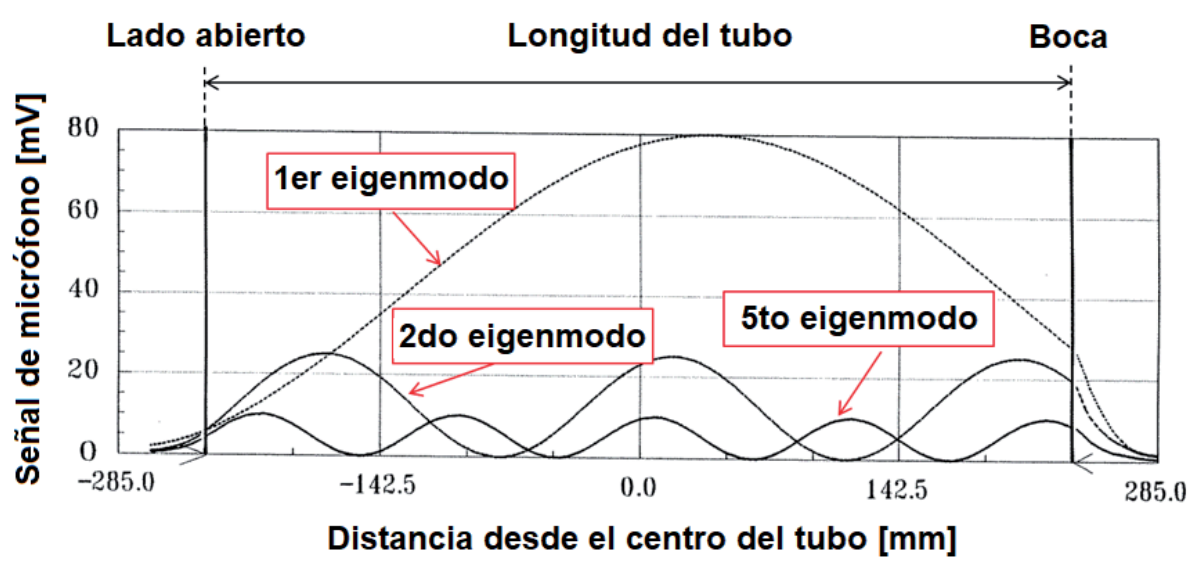

Fig. 6. Ondas de pie en un tubo de órgano. Se muestran las distribuciones de presión sonora del primer, tercer y quinto eigenmodo en un tubo ancho. 
Estos hechos experimentales pueden comprenderse teniendo en cuenta las propiedades físicas de los tubos del órgano como un resonador acústico. La columna de aire en los tubos tiene varios modos propios (patrones de onda estacionaria) con frecuencias de resonancia características (eigenfrecuencias). Sus frecuencias no están relacionadas armónicamente debido a la corrección final, ${ }^{12}$ que disminuye con la frecuencia. ${ }^{2}$ Dado que la corrección final es proporcional al diámetro del tubo, el estiramiento de las eigenfrecuencias es mayor para los tubos anchos que para los estrechos. Además, la corrección final para una pequeña abertura (boca) es más grande que la del extremo abierto más grande. Por lo tanto, el estiramiento de la eigenfrequencia de un tubo de órgano es más grande para tubos anchos que angostos de la misma longitud y diámetro. Debido a la diferentes correcciones finales en las aberturas, la onda estacionaria se encuentra asimétricamente dentro del tubo del órgano. ${ }^{13}$ Por lo tanto, el espectro de sonido en la boca y en el extremo abierto son diferentes, como se muestra en la figura 4.

\section{Una línea de base dependiente de la frecuencia}

Se determina la línea de base del espectro (véase la figura 4) por el ruido de banda ancha en la boca del tubo. Este ruido es producido por el flujo de aire en la salida y la parte superior labio. ${ }^{14}$ Dado que el resonador amplifica ese ruido alrededor de las eigenresonancias, el ruido amplificado puede dominar el sonido del tubo en el rango de alta frecuencia, donde las parciales del armónico fundamental son generalmente débiles. El ruido de alta frecuencia puede reducirse muy eficazmente cortando, por ejemplo, las ranuras de corte en el lengüeta (placa de metal separando el pie y el cuerpo del tubo; figura 2. ${ }^{15}$ Este método puede aumentar la proporción de la parciales armónicas a la línea de base significativamente.

\section{Envoltura de las armónicas parciales}

La forma de la envoltura depende de las pérdidas totales en el tubo que incluyen las pérdidas de volumen en el aire, la pérdida superficial en la pared de los tubos debido a la viscosidad y la conducción del calor, las pérdidas de radiación en las aberturas, y la pérdida de energía en el acoplamiento del sonido con las vibraciones de la pared. Para los tubos del órgano, la superficie y las pérdidas de radiación son mucho mayores que los otros dos efectos. A la misma frecuencia, la pérdidas superficiales son relativamente mayores y las pérdidas por radiación son relativamente menores para tubos estrechos que para tubos anchos. Ya que las pérdidas superficiales disminuyen y las pérdidas de radiación aumentan con la frecuencia, la pérdida mínima se produce a una cierta frecuencia. De hecho, tal mínimo se puede observar en tubos estrechos. Visto de otra forma, la mayor amplitud no se produce para la fundamental, pero para una parcial superior. La medición del espectro de un tubo normal, ancho, y estrecho se muestra en la figura 7. En el caso de tubo resonador ancho/estrecho hay menos/más parciales, respectivamente, que para el resonador normal de la tubería.

Las pérdidas de radiación se producen a través de la radiación acústica en las aberturas de los tubos (boca y extremo abierto). Debido a que las aberturas son 

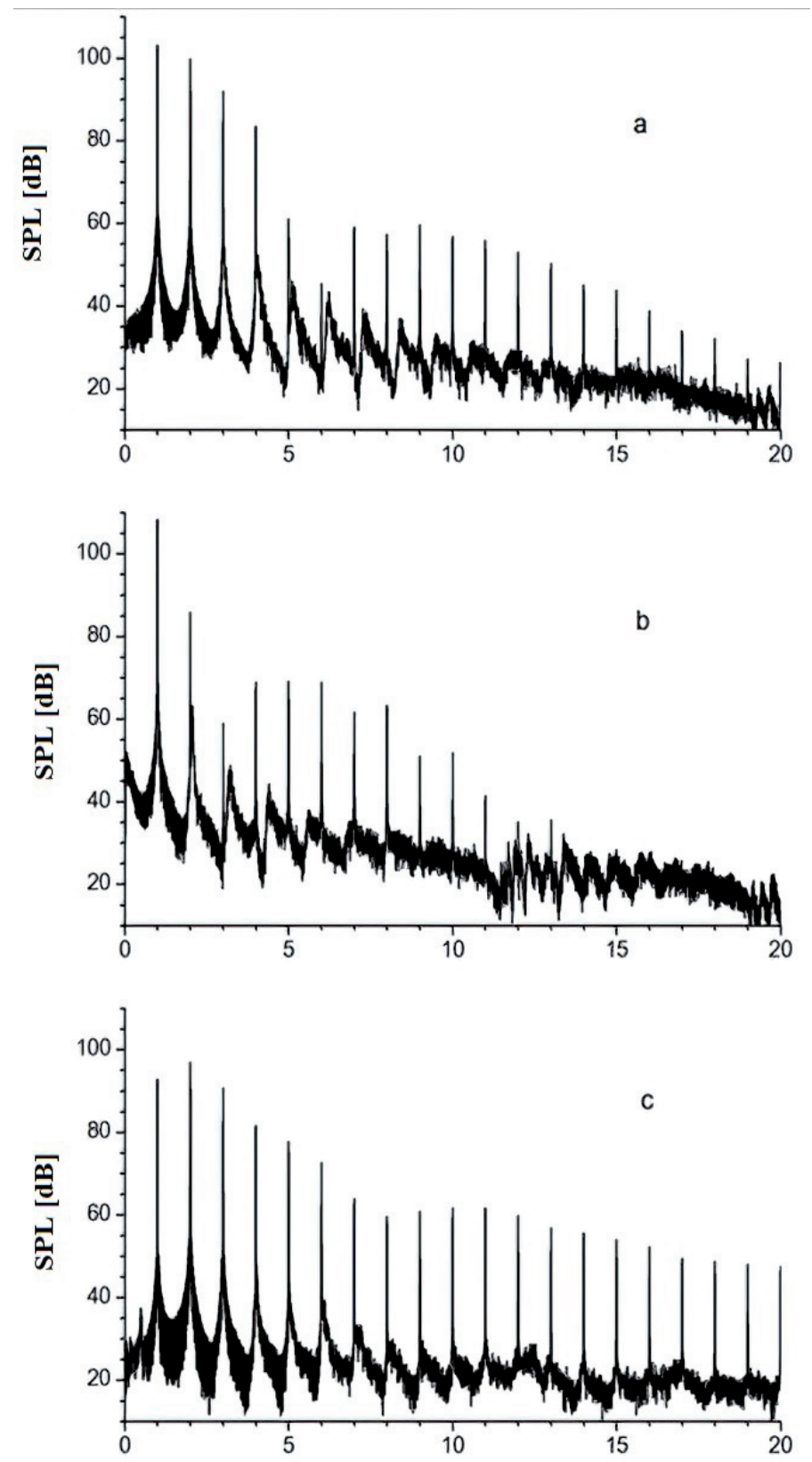

Fig. 7. Espectros medidos de un Diapasón (normal; a), una Flauta (ancha; b), y un tubo Salicional (estrecho; c).

mucho menores que la longitud de onda del sonido, ambas pueden considerarse fuentes simples (monopolos). ${ }^{13}$ Mediciones mediante un sistema de cámara acústicas confirman este modelo de fuente simple. ${ }^{16}$ La figura 8 muestra que las fuentes del sonido son realmente las aberturas en la boca y en el extremo abierto de la tubo. Basado en el registro de la primera parcial (fundamental) en la figura $8 \mathrm{a}$ (véase http://acousticstoday.org/8a.mp4) el sonido se irradia en fase pero con diferente intensidad. La presión acústica es mayor en la boca. La figura $8 \mathrm{~b}$ (ver http:// acousticstoday.org/8b.mp4) muestra que las dos fuentes irradian en fase opuesta. La fuente simple en la boca es generalmente mucho más fuerte que la fuente en el extremo abierto. 


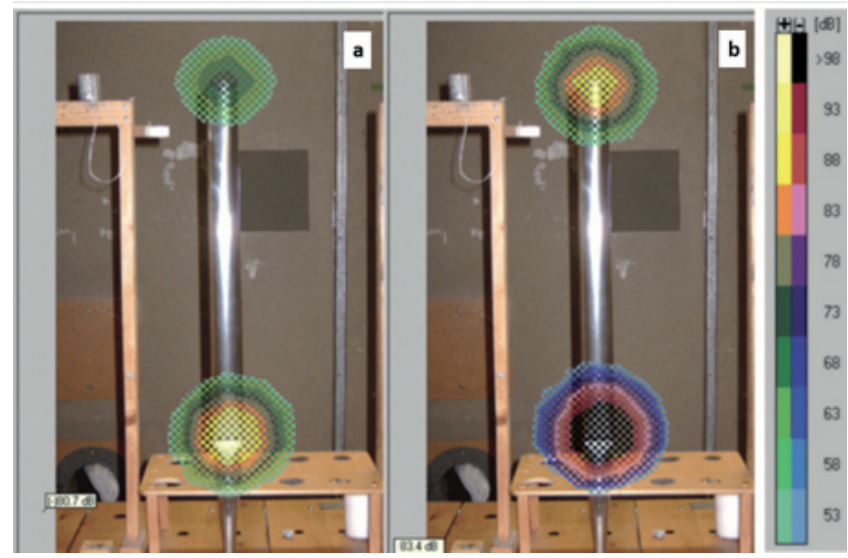

Fig. 8. Radiación sonora en las partes primera (a) y segunda (b). Los colores corresponden a la intensidad del sonido. (véase http://acousticstoday.org/8a.mp4 y http:// acousticstoday.org/8b.mp4).

La envolvente de los armónicos del espectro sonoro en la boca presenta una estructura de forma con un mínimo visible (ver figuras $4 \mathrm{~b}$ y 7 ) debido a la posición relativa de las armónicas parciales y los eigenmodos vecinos. Debido al estiramiento de las frecuencias propias, las partículas armónicas se desplazan gradualmente de los picos de los eigenmodos en el valle entre ellos y luego más lejos hacia el pico del eigenmodo vecino inferior. Si el armónico está cerca de la eigenfrecuencia, el armónico parcial será amplificado por la eigenresonancia. Un armónico parcial que cae a medio camino entre dos modos no será amplificado mientras que el armónico parcial más cercano al mínimo entre dos eigenmodos será el más pequeño. Por lo tanto, se puede observar un formante mínimo en los espectros medidos en la boca. Debido a que el estiramiento es más pronunciado para tubos más anchos, la posición del formante mínimo depende de la relación diámetro-longitud del tubo.

Espectros de sonido medidos en la boca de un tubo normal (Diapasón), ancho (flauta), y estrecho (Salicional o de cuerda) muestran claramente este efecto (figura 7). Para el tubo Diapasón, el primer mínimo se encuentra en la sexta parcial (figura 7a). Para el tubo de flauta, el mínimo se produce alrededor del tercer parcial (figura 7b); para el tubo Salicional se desplaza hasta el octavo parcial (figura $7 \mathrm{c}$ ).

Diferentes cubiertas espectrales en la boca y en el extremo abierto

Se ha demostrado que el campo acústico radiado corresponde al de dos fuentes simples situadas en las aberturas de los tubos (ver figura 8). Las fuentes simples irradian en fase para los armónicos parciales impares y fuera de fase para los pares. La fuerza es diferente para ambas fuentes, y el dos aberturas irradian diferentes espectros. ${ }^{13}$ Los espectros del sonido irradiado en las aberturas es diferente porque las ondas estacionarias en el tubo están asimétricamente localizadas (ver figuras 4 y 6). Porque el extremo corrección es inversamente proporcional al área de la abertura, ${ }^{13}$ la envoltura mínima se produce para el armónico parcial inferior en la boca que en el extremo abierto. Es decir, los envoltorios espectrales en la boca y el extremo abierto siempre son diferentes. 


\section{IRREGULARIDADES EN LA ALTA FRECUENCIA Parte del espectro}

Se pueden causar irregularidades en el rango de armónicos superiores por la excitación de resonancias transversales (corte transversal eigenmodos) del tubo. Las filas de tubos pueden tener armónicos parciales en el rango de resonancias transversales; por lo tanto, pueden aparecer resonancias transversales en el espectro entre los armónicas parciales (figura $7 \mathrm{~b}$, primera resonancia transversal alrededor del parcial undécimo). Estas resonancias se excitan por el ruido de alta frecuencia en el labio superior.

Las irregularidades en el espectro también pueden ser causadas por las vibraciones de la pared. Se ha demostrado que las vibraciones de la pared no pueden irradiar sonido directamente. ${ }^{17,18}$ Por otra parte, existe un acoplamiento lineal entre la columna de aire y la pared del tubo para tubos rectangulares ${ }^{16} \mathrm{y}$ también para tubos cilíndricos si la sección transversal del tubo no es un círculo perfecto, pero es ligeramente elíptica o la pared es muy delgada. ${ }^{19}$ En estos casos, las vibraciones de la pared pueden influir en el sonido irradiado en las aberturas, especialmente durante el transitorio. ${ }^{16,19} \mathrm{Si}$ un modo de vibración aguda está cerca de un eigenmodo o armónica parcial del sonido de los tubos, ambos modos estarán acoplados, lo que lleva a un ligero desentono del componente de sonido correspondiente. Sin embargo, tal coincidencia es muy rara en la práctica. La figura 9 muestra los diagramas de vibración registrados por un vibrómetro láser tridimensional (3-D) de un par de tubos Diapason G. La figura 9a (http:// acousticstoday.org/9a.mp4) muestra los tubos de metal liso (aleación de estañoplomo) en el quinto parcial $(974 \mathrm{~Hz}$ ) y la figura $9 \mathrm{~b}$ (http://acousticstoday.org/9b. mp4) muestra la tuberías de zinc en el quinto parcial (misma frecuencia). Es evidente que a pesar de la misma frecuencia de medición, los tubos hechos de diferentes materiales muestran muy diferente modo de vibración.

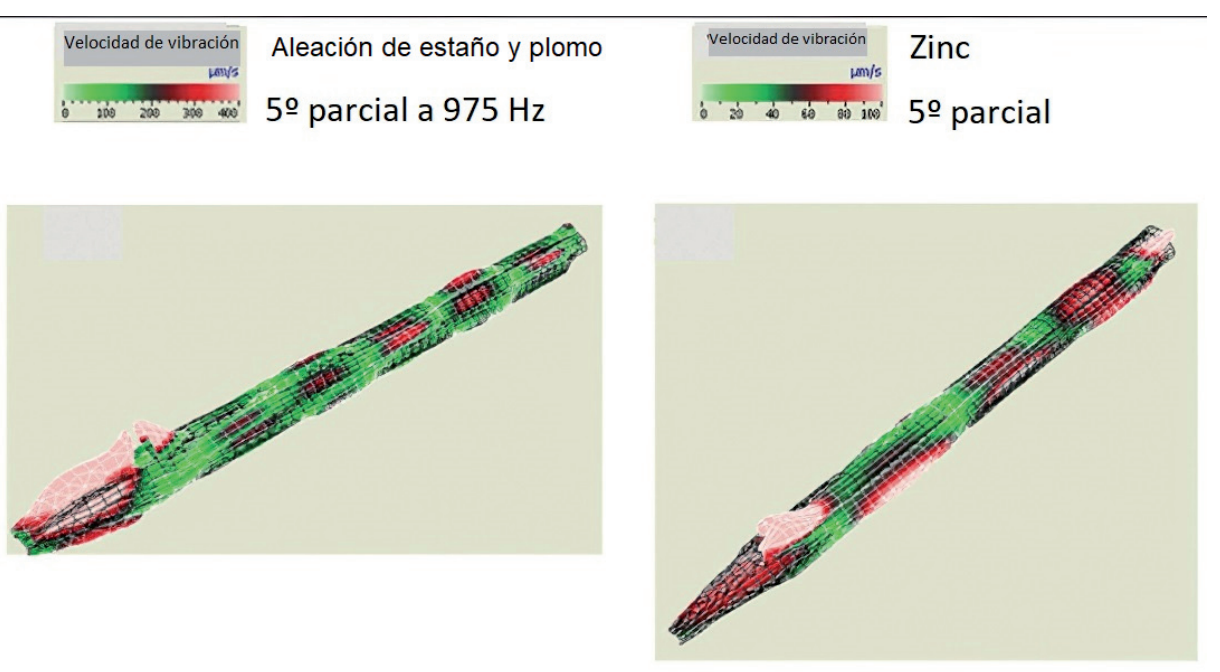

Fig. 9. Forma de vibración del par de tubos Diapason G. Rojo indica que las paredes del tubo son vibradas duro y verde significa menos vibración. a: Tubo de metal liso (aleación de estaño-plomo) en la quinta parte parcial $(974 \mathrm{~Hz}$, véase http://acousticstoday. org/9a.mp4). b: Tubo de zinc en el quinto parcial (y la misma frecuencia, véase http:// acousticstoday.org/9b.mp4). 
La figura 10 muestra la representación 3-D de la ataques transitorios (ataque; cómo se construirán las parciales en tiempo) del mismo par de tubos Diapasón G (mostrado hasta el sexto parcial), y los ruidos entre las parciales también se registran. Es obvio que los ataques de los dos tubos son muy similares. Los experimentos mostraron que las diferencias en los ataques registrados con tubos hechos de diferentes materiales no son más grandes que con dos ataques sucesivos de los mismos tubos.

Las explicaciones anteriores demuestran que los mecanismos influyen en el sonido constante de las tuberías. Por otra parte, incluso las características más complicadas podrían asignarse a las propiedades físicas medibles y comprensibles de los tubos de órganos de combustión. Los resultados de la investigación en acústica de tubos de salida se pueden adaptar en la investigación aplicada para apoyar el trabajo diario de los órganos constructores. La siguiente sección presenta un ejemplo de las tendencias en la investigación aplicada.
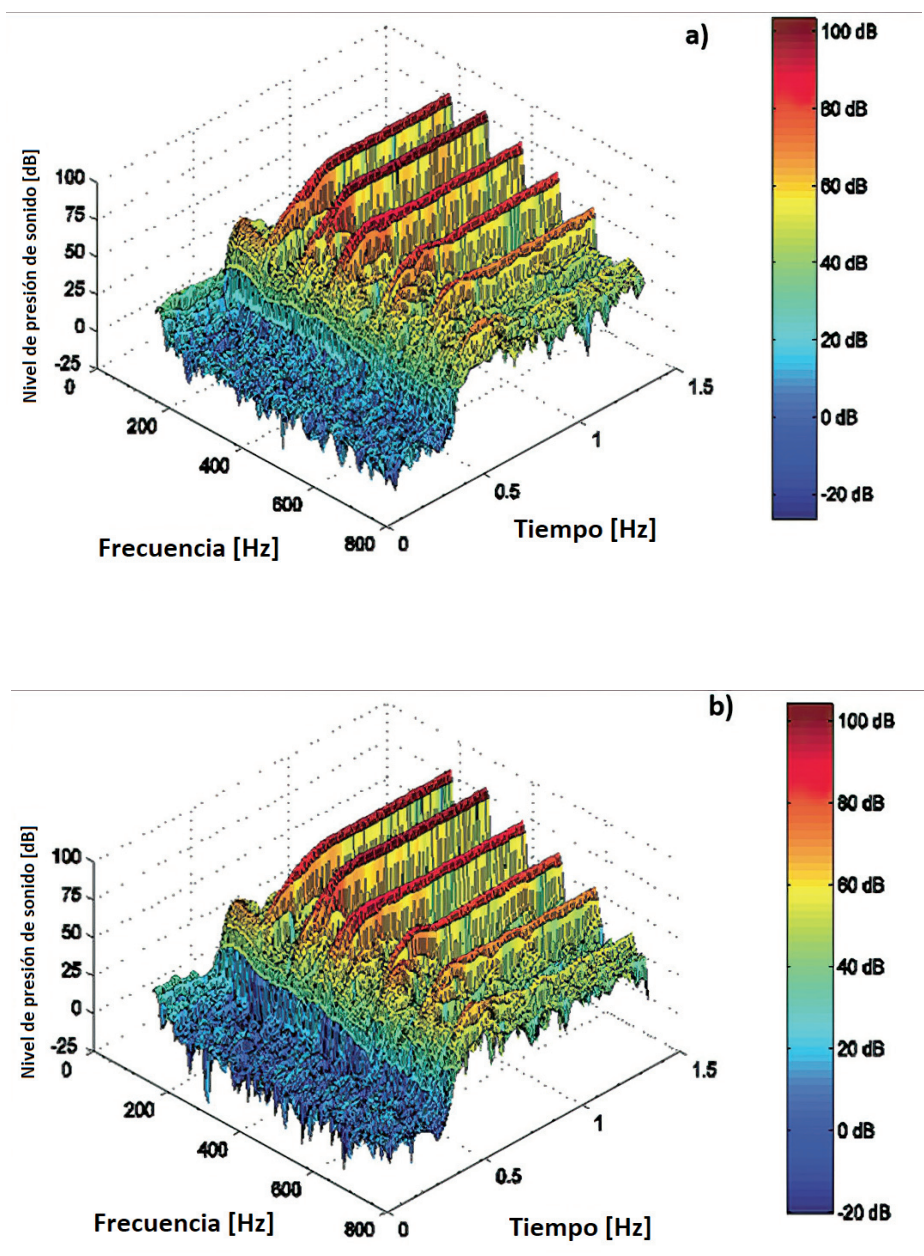

Fig. 10. Una representación en 3-D de los transitorios de ataque analizados del par de tubos Diapasón $\mathrm{G}$ (igual que se muestra en la Figura 9). El gráfico muestra el inicio del sonido de la tubería y, por lo tanto, cómo las partes parciales de las diferentes frecuencias se acumulan en el tiempo. a: Hecho de metal liso (aleación de estaño-plomo). b: Hecho de zinc.mp4). b: Tubo de zinc en el quinto parcial (y la misma frecuencia, véase http://acousticstoday.org/9b.mp4). 


\section{Diseño sonoro de tubos}

Los tubos de órgano tipo flauta son semiabiertos cuyo resonador consiste en dos partes principales: una recta principal cilíndrica y una libre más corta y más delgada unida a su parte superior (figura 11, arriba a la izquierda). La longitud y el diámetro puede variar, y esto hace posible que el ajustar el timbre. Por ejemplo, os tubos del los órganos del estilo barroco deben tener un sonido rico en el quinto (tercer armónico) puro, mientras que los instrumentos de estilo romántico requieren más de una tercera mayor (quinto armónico) en el sonido. Para poder para cumplir estos requisitos, se necesitan reglas especiales de diseño para determinar las dimensiones de los tubos de manera que se pueda alcanzar el carácter deseado del sonido. El proceso de determinar las dimensiones geométricas apropiadas de los tubos de órganos con el fin de lograr un timbre predefinido se conoce como diseño sonoro.

La construcción del tubo de desfogue fue estudiado por diferentes investigadores, sobre todo Helmholtz. Su conclusión fue que para reforzar el quinto armónico en el sonido, lo mejor es tener un desfogue de una longitud de dos quintos de la del resonador principal. ${ }^{20} \mathrm{Al}$ parecer, en un examen más reciente, la configuración propuesta por Helmholtz resultó ser el menos favorable. ${ }^{21}$ En el estudio iniciado por los constructores de órganos y realizado por los autores de este artículo, se estableció una novedosa metodología para el diseño sonoro de tubos y se implementó en una herramienta de software.
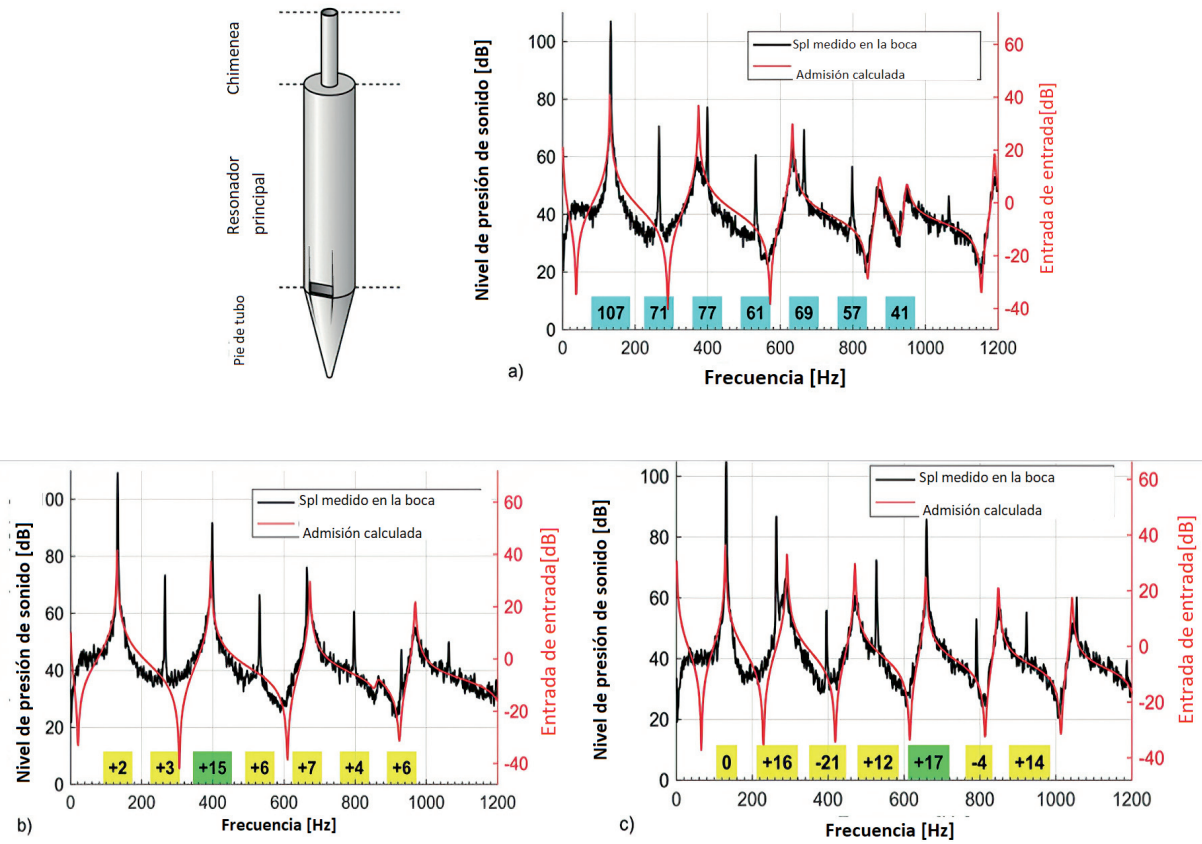

Fig. 11. Arriba a la izquierda: bosquejo de un tubo de órgano de chimenea. a ç c: Espectros medidos (negro) y entrada calculada (rojo) de las tuberías de chimenea experimentales. a: Diseño de la pipa de chimenea de referencia. Los números en el fondo azul son las amplitudes de las primeras siete parciales armónicas. b: Diseño optimizado que mejora el tercer armónico (quinto puro) por $15 \mathrm{~dB}$. c: Diseño optimizado que mejora el quinto (tercer mayor) por $17 \mathrm{~dB}$. Los números en los fondos verde y amarillo muestran los niveles relativos de los armónicos comparados con el tubo de referencia. 
La idea del enfoque de diseño sonoro propuesto es afinar las frecuencias propias del resonador para que coincidan con las frecuencias de los armónicos parciales predefinidos del sonido. ${ }^{22}$ Cuando un armónico parcial se traslapa con una eigenfrecuencia, el eigenmodo correspondiente consigue excitarse muy eficientemente y por lo tanto se puede esperar la amplificación del armónico. Mediante simulación por computadora, se calcula la llamada admisión de entrada. Los picos de la admisión de entrada corresponden a los picos de eigenresonancias. Es importante que los picos de las curvas rojas en la figura 11 coincidan con la armónica parcial a ser mejorada. Los espectros sonoros medidos en estado estacionario se muestran en figura 11a-c. En cada diagrama, la presión acústica medida en la boca del tubo y la admisión de entrada calculada se muestran por las líneas negras y rojas, respectivamente. La figura 11a muestra el tubo de referencia con la amplitud de la primeros siete armónicos, indicados por los números en el fondo azul. El tubo de referencia tiene un componente fundamental fuerte en su sonido mientras que los armónicos superiores son muy débiles. La figura $11 \mathrm{~b}, \mathrm{c}$ muestra los resultados optimizados para los armónicos tercero y quinto, respectivamente. Los números en el fondo verde indican la amplificación del objetivo armónico parcial en comparación con los niveles medidos en el caso del tubo de referencia. Los números en el fondo amarillo muestran los mismos cambios en los niveles de los otros armónicos. Como se puede ver, los resonadores optimizados pueden mejorar los armónicos dirigidos por más de $15 \mathrm{~dB}$ manteniendo la frecuencia fundamental constante. Esta amplificación puede considerarse sustancial si se tiene en cuenta que los tubos experimentales sólo diferían en la geometría de sus resonadores.

\section{CONCLUSIONES}

La intención de los autores de este trabajo fue demostrar que la investigación sobre tubos de órganos conduce a una mejor comprensión de cómo funcionan. Además, la investigación puede proporcionar explicaciones científicas para apoyar o refutar creencias establecidas de los constructores de órganos y, por último pero no menos importante, puede proporcionar nuevos resultados científicos y herramientas para mejorar aún más el arte de la construcción de órganos de tubo.

\section{REFERENCIAS}

1. Miklós, A., and Angster, J. (2000). Properties of the sound of flue organ pipes. Acta Acustica united with Acustica 58, 611-622.

2. Fletcher, N. H., and Rossing, T. D. (1991). The Physics of Musical Instruments. Springer-Verlag, New York.

3. Miklós, A., Angster, J., Pitsch, S., and Rossing, T. D. (2003). Reed vibration in lingual organ pipes without the resonators. The Journal of the Acoustical Society of America 113, 1081-1091.

4. Miklos, A., Angster, J., Pitsch, S., and Rossing, T. D. (2006). Interaction of reed and resonator by sound generation in a reed organ pipe. The Journal of the Acoustical Society of America 119, 3121-3129.

5. Yoshikawa, S., and Saneyoshi, J. (1980). Feedback excitation mechanism in organ pipes. The Journal of the Acoustical Society of Japan (E) 1, 175-191. 
6. Verge, M. P., Fabre, B., Mahu, W. E., and Hirschberg, A. (1994). Feedback excitation mechanism in organ pipes. The Journal of the Acoustical Society of America 95, 1119-1132.

7. Fletcher, N. H. (1996). Sound production by organ flue pipes. The Journal of the Acoustical Society of America 60, 926-936.

8. Töpfer, J. G. (1888). Die Theory und Praxis des Orgelbaues. Zweites Buch, Voigt, Weimar, Germany, pp. 71-83.

9. Mahrenholz, C. (1987). Berechnung der Mensuren. Orgelbau Fachverlag Rensch, Lauffen am Neckar, Germany, Chap. B., pp. 35-125.

10.Angster, J., and Miklós, A. (1995). Documentation of the sound of a historical pipe organ. Applied Acoustics 46, 61-82.

11.Korn, G. A., and Korn T. M. (1975). Mathematical Handbook for Scientists and Engineers. McGraw-Hill, New York, Chap. 4.11.4, pp. 140-142.

12.Nelkon, M., and Parker, P. (1970). Advanced Level Physics. Heinemann Educational Books Ltd., London, pp. 640-674.

13.Angster, J., and Miklós, A. (1998). Sound radiation of open labial organ pipes; The effect of the size of the openings on the formant structure. In Proceedings of the International Symposium on Musical Acoustics (ISMA), Leavenworth, WA, June 26 to July 1, 1998, pp. 267-272.

14.Fabre, B., Hirschberg, A., and Wijnands, A. P. J. (1996). Vortex shedding in steady oscillation of a flue organ pipe. Acta Acustica united with Acustica 82, 863-877.

15.Angster, J., Paál, G., Garen, W., and Miklós, A. (1997). Effect of voicing steps on the stationary spectrum and attack transient of a flue organ pipe. Proceedings Institute of Acoustics 19, 285-294.

16.Angster, J., Dubovski, Z., Pitsch, S., and Miklos, A. (2011). Impact of the material on the sound of flue organ pipes (Acoustic and vibration investigations with modern measuring techniques). In Birnbaum, C. (Ed.), Analysis and Description of Music Instruments Using Engineering Methods. Stiftung Händel-Haus (Handel House Foundation), Halle (Saale), Germany, pp. 3441.

17.Backus, J., and Hundley, T. C. (1965). Wall vibrations in flue organ pipes and their effect on tone. The Journal of the Acoustical Society of America 39, 936-945.

18.Angster, J., Paál, G., Garen, W., and Miklós, A. (1998). The effect of wall vibrations on the timbre of organ pipes. In Proceedings of the 16th International Congress on Acoustics and 135th Acoustical Society of America Meet ing, Seattle, WA, June 20-26, 1998, pp. 753-754.

19.Kob, M. (2000). Influence of wall vibrations on the transient sound of flue organ pipes. Acta Acustica united with Acustica 86, 642-648.

20.Helmholtz, H. (1954). On the Sensation of Tone. Dover, New York.

21.Kokkelmans, S. J. J. M. F., Verge, M.-P., Hirschberg, A., Wijnands, A. P. J., and Schoffelen, R. L. M. (1999). Acoustic behavior of chimney pipes. The Journal of the Acoustical Society of America 105, 546-551.

22.Rucz, P., Trommer, T., Angster, J., Miklós, A., and Augusztinovicz, F. (2013). Sound design of chimney pipes by optimization of their resonators. The Journal of the Acoustical Society of America 133, 529-537. 